\title{
Uptake of macromolecules by cercariae during skin penetration and transformation to schistosomula (Schistosoma mansoni)
}

\author{
Joyce Thornhill' ${ }^{1}$ John Kusel ${ }^{1 /+}$, Fabricia Alvisi de Oliviera'², Fabio Ribeiro ${ }^{2}$, \\ Symone Fulgêncio Lima², Paulo Marcos Zech Coelho², Paul McVeigh ${ }^{3}$, Ana Carolina Alves Mattos \\ 'University of Glasgow, GBRC 120 University Place, G12 8TA Glasgow, Scotland, UK ²Instituto de Pesquisas René Rachou, \\ Belo Horizonte, MG, Brasil ${ }^{3}$ Queen's University Belfast, Belfast, Northern Ireland, UK
}

Here, we observed the uptake of membrane-impermeant molecules by cercariae as they penetrate the skin and are transformed into schistosomula. We propose that membrane-impermeant molecules, Lucifer Yellow, Propidium iodide and Hoechst 33258 enter the parasite through both thenephridiopore and the surface membrane and then diffuse throughout the body of the parasite. We present a hypothesis that the internal cells of the body of the schistosomulum represent a new host-parasite interface, at which skin-derived growth factors may stimulate receptors on internal membranes during transformation of the cercariae into the schistosomulum.

Key words: Schistosoma mansoni - membrane impermeant Lucifer Yellow - nephridiopore - host/parasite interface - linoleic acid

The penetration of mammalian skin by cercariae of Schistosoma mansoni is accompanied by many changes in the structure and metabolism of the parasite (McLaren \& Hockley 1976, Skelly et al. 1993, Bahia et al. 2007). A very dramatic change is the loss of the tail via its severance from the body to yield the schistosomulum (Stirewalt et al. 1966, Howells et al. 1975, McLaren \& Hockley 1976). During the tail loss, the nephridiopore (the aperture representing the external exit of the excretory system) is fully exposed to the external environment. Recently, a pathway was described that involves the entry of large molecules into the body through the nephridiopore after surface membrane damage (Tan et al. 2003). In the present paper we describe the process of uptake of membrane-impermeable fluorescent dyes by the cercariae as they penetrate the skin and we propose that these molecules may enter through both the nephridiopore and the surface membrane.

\section{MATERIALS AND METHODS}

Fluorescent chemicals - Lucifer Yellow CH lithium and potassium salt, Hoechst 33258, Propidium iodide and Texas Red bovine serum albumin were purchased from Invitrogen (UK). FITC-labelled dextrans with molecular weights of 4,10 and $70 \mathrm{kDa}$ were purchased from Sigma (UK). All fluorescent chemicals are water soluble and were dissolved (1 mg per mL) in RPMI-1640.

Origin of parasite material, skin penetration and in vitro culture - A Puerto Rican isolate of $S$. mansoni was maintained in our laboratory in Biomphalaria glabrata and the outbred TO strain of mice (obtained from Glas-

\footnotetext{
+ Corresponding author: johnkusel@hotmail.com

Received 12 November 2008

Accepted 4 September 2009
}

gow University). Skin schistosomula were obtained by allowing cercariae, which were concentrated by light, to penetrate into a shaved area of a recently euthanised mouse. Mice were euthanised by cervical dislocation and their abdomens were shaved with an electric shaver. Two hundred-microlitre intradermal injections containing $1 \mathrm{mg}$ per $\mathrm{mL}$ of fluorescent dye were delivered $(26 \mathrm{G}$ needle) and flanged open tubes were placed on the skin. The flanges were pressed onto the skin surface. One millilitre of cercarial suspension was gently poured through the open end of tube and, in this way, the cercariae could contact the skin in the region labelled with the dye. After $15 \mathrm{~min}$, the skin region exposed to the cercariae was removed with scissors, cut into small pieces and incubated for $2 \mathrm{~h}$ in RPMI-1640 supplemented with $10 \%$ foetal calf serum at $37^{\circ} \mathrm{C}$.

Production of schistosomula in vitro - Schistosomula were produced in vitro according to the method of Colley and Wikel (1974). Cercariae were suspended in RPMI1640 during the syringe transformation.

Fixation of cercariae - Labelled schistosomula were fixed for $4 \mathrm{~h}$ with $4 \%$ paraformaldehyde. The $4 \%$ paraformaldehyde was prepared by dissolving the solid paraformaldehyde (Sigma-Aldrich, UK) in RPMI-1640 at $60^{\circ} \mathrm{C}$.

Examination of parasites by fluorescence microscopy - Photographs were taken after observation of the parasites under a 40X objective lens of a Leica Laborlux $\mathrm{S}$ microscope. All labelled parasites were viewed and photographed using a Leitz Orthoplan Laborlux S microscope with a Leica camera attachment. This allowed the variability in the labelling of the parasite population to be observed, counted and recorded. Photographs were taken after observation of the parasites under the $40 \mathrm{X}$ or $50 \mathrm{X}$ objectives. Further detail in the distribution of the labelled compounds was obtained using a Zeiss microscope (Axioplan 2). Confocal microscopy was carried out with a Leica TCS NT AOBS SP2 confocal microscope. 


\section{RESULTS}

In vivo labelling during penetration of fluorescentlyinfiltrated mouse skin - Schistosomula were recovered from the skin labelled with fluorescent dyes (Lucifer Yellow and Propidium iodide) after an incubation of $30 \mathrm{~min}$ or $2 \mathrm{~h}$. The worms were then examined by fluorescence microscopy (Fig. 1). Fig. 1 shows that Lucifer Yellow ( 0.45 $\mathrm{kDa}$ ) clearly labelled the subtegumental area close to the excretory tubules, as well as some other organs (e.g., the gut and the nephridiopore). Fig. 1 summarises the results at both $30 \mathrm{~min}$ and $2 \mathrm{~h}$. A similar pattern is observed when fluorescent dextrans with molecular weights of 3-20 kDa were used in the skin assay (results not shown).

Transformation of cercariae into schistosomula by syringe passage - Cercariae were syringe transformed into schistosomula in the presence of each of the dyes. A subsequent incubation at $37^{\circ} \mathrm{C}$ in RPMI-1640 showed that, although some of the schistosomula incubated in Lucifer Yellow appeared very similar to the skin transformed schistosomula (Fig. 1), the results were much more variable for these mechanically transformed forms and only a proportion of the parasites had taken up the dye to provide a clear internal localisation. This result is illustrated in Fig. 2, which shows that many of the schistosomula were diffusely fluorescent, without the clear definition of organelles and tissues apparent in Fig. 1. In Figs 1 and 2, free swimming cercariae were incubated at RT with Lucifer Yellow for 30 min and washed three times in RPMI-1640 as a negative control. Some Lucifer Yellow adhered to the surface and very small amounts entered the body, providing a very diffuse pattern unlike the definite structures observed in Fig. 1. In a few specimens, the fine outline of the excretory tubules could be seen. Any internal Lucifer Yellow was excreted from these parasites (data not shown).

Specific stimulus from skin - The variability in the amount of fluorescent label (Lucifer Yellow and Propidium iodide) taken up by the cercariae appeared to be much less in those parasites recovered from the skin after $30 \mathrm{~min}$ or $2 \mathrm{~h}$, whereas in vitro-transformed schistosomula showed considerable differences in uptake between individual parasites. These differences were not genetically determined since three clones of cercariae, produced from monomiracidial infections of snails, also displayed this variability. When the brightly labelled parasites (such as that seen in Fig. 2) were counted and expressed as a percentage of the total population after transformation in the presence of the dyes, the values obtained for the three clones were $11 \%, 14 \%$ and $24 \%$. The value obtained for a mixed population of cercariae (from a routine multimiracidial infection) was $17 \%$. We argue here that there must be a specific stimulus from the skin penetration process that was lacking in the in vitro transformation process. The composition of the medium used during syringe transformation was varied in an attempt to reduce the differences in uptake between parasites formed in vitro and those formed on the skin. Uptake occurred to the same extent (between $10-25 \%$ brightly labelled parasites) in RPMI-1640, sodium chloride $(0.15 \mathrm{M})$, potassium chloride $(0.15 \mathrm{M})$ and choline chloride $(0.15 \mathrm{M})$, but it was inhibited ( $5 \%$ brightly labelled parasites) in glucose $(0.2 \mathrm{M}$

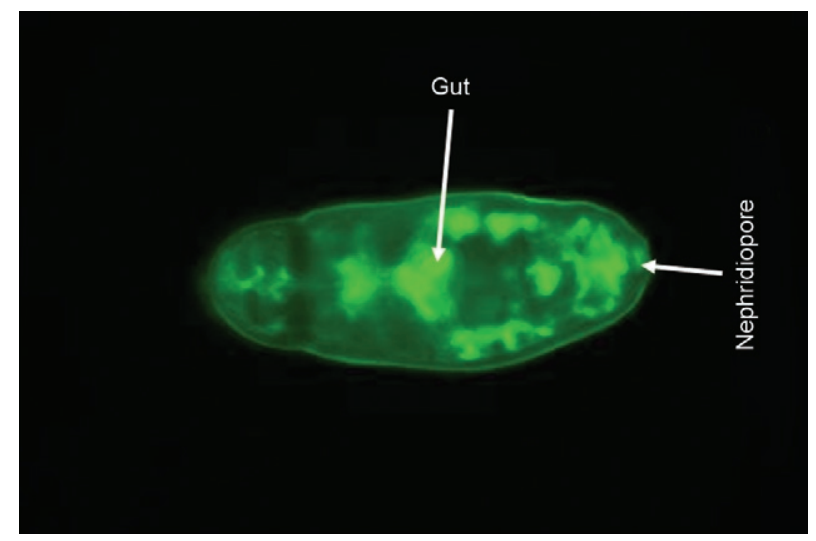

Fig. 1: cercariae were applied to mouse skin into which membrane impermeant Lucifer Yellow, Propidium iodide and Hoechst 33258 had been injected. The schistosomulum shown had been recovered from the incubation medium after excision of skin and incubation of fragmented skin in RPMI-1640 plus foetal calf serum at $37^{\circ} \mathrm{C}$ for $2 \mathrm{~h}$. All dyes are taken up and Lucifer Yellow is shown here by fluorescent microscopy. The dye is internal and localised around the nephridiopore and the subtegumental region near the excretory tubules. Bar $=50 \mu \mathrm{m}$.

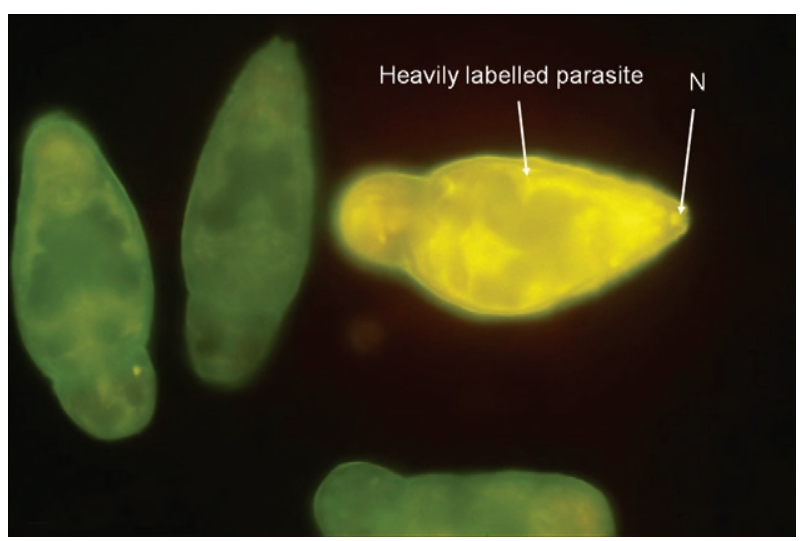

Fig. 2: cercariae were transformed in vitro by the method of Colley and Wikel (1974). In the presence of the dyes described in Fig. 1. A proportion of the schistosomula (about $20 \%$ ) take up the dyes to give heavy labelling, but the remainder show only minimal uptake of the dyes. $\mathrm{Bar}=50 \mu \mathrm{m}$.

in water) and in water alone. From these results, we concluded that some kind of charged molecule must be present in the transformation fluid to allow uptake to occur. When the cercariae were transformed in $50 \%$ foetal calf serum in RPMI-1640, the percentage of parasites that were brightly labelled increased to $60 \%$. Thus, foetal calf serum may contain a compound that stimulates uptake. We conclude that there is a specific, but as yet unknown, stimulus provided to the cercariae from the skin that enables the cercariae to open the nephridiopore or alter the surface membrane permeability and allow the uptake of molecules.

Nature of the skin stimulus - The work of Haas et al. $(1997,2002)$ and Grabe and Haas (2004) provided insight as to the nature of the skin stimulus. At the epidermal surface, arginine, ceramides and linoleic acid stimulate 


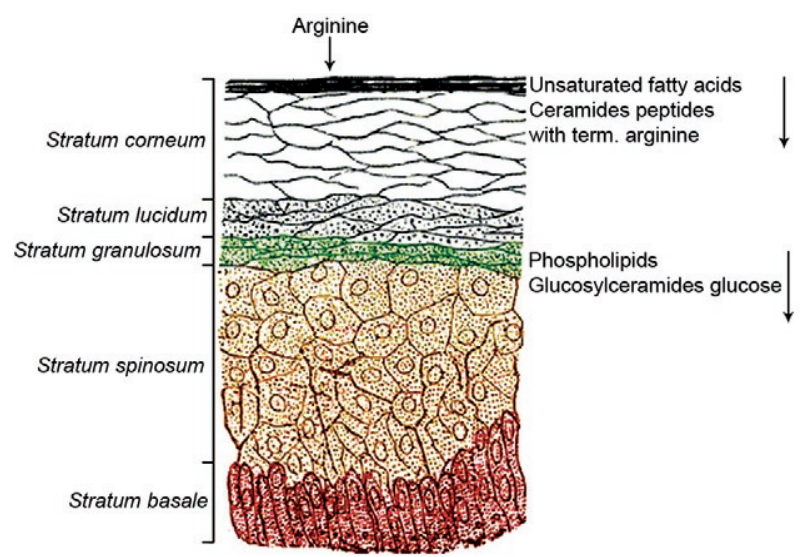

Fig. 3: diagram of the structure of human skin showing the location of the molecules (arginine, linoleic acid, glucosylceramides, glucose) found by Haas et al. (e.g. Haas et al. 2002) to be important in the migration of schistosomula in the skin after cercarial penetration.

the parasite, while phospholipids, glucosyl ceramides and glucose are the major stimuli deeper in the epidermis (Fig. 3). Two hours after the transformation, linoleic acid $(0.5 \mathrm{mM})$ was added to the schistosomula for a period of $15 \mathrm{~min}$ in the presence of Lucifer Yellow. The results showed a highly significant increase in the number of brightly fluorescent schistosomula which showed uptake of the fluorescent dyes (in a mean of 6 experiments), with an increase from $16 \%$ brightly labelled parasites (without linoleic acid) to $75 \%$ brightly labelled parasites in the presence of linoleic acid. Whether this uptake occurs through the nephridiopore or through the surface membrane has yet to be determined.

Evidence that uptake of fluorescent dyes might occur through the nephridiopore - Cercariae transformed in vitro in the presence of Lucifer Yellow were fixed in paraformaldehyde and examined by confocal microscopy. In the brightly labelled parasites, the major area of dye localisation was near or in the nephridiopore (Fig. 4). However, in many images, the surface membrane was also labelled. Thus, uptake may also occur through the surface membrane. Cercariae were incubated with Propidium iodide and Hoechst 33258 at RT in vitro and watched under a fluorescence microscope. Over a period of $20 \mathrm{~min}$, the dyes were initially observed at the nephridiopore and they then could be seen to diffuse into the body of the cercaria and follow a pathway adjacent to the excretory tubules (Fig. 5). Since these experiments were carried out at RT, we cannot exclude the possibility that the region of the surface membrane near the nephridiopore is permeable to these dyes.

\section{DISCUSSION}

In this paper, we report that, during skin penetration or syringe transformation of cercariae, membrane-impermeant molecules such as Propidium iodide and Lucifer Yellow can enter the body of the parasite. FITC dextrans (3-20 kDa) were also observed to enter the body of the
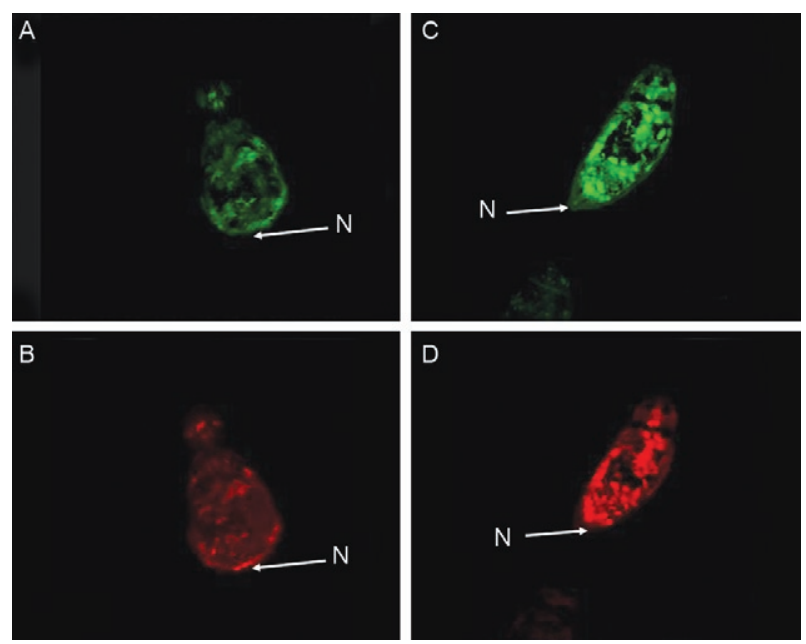

Fig. 4: confocal microscopy of paraformaldehyde fixed schistosomula immediately after in vitro transformation in the presence of Lucifer Yellow (A, C) and Propidium iodide (B, D). The schistosomula show a high concentration of dyes near the nephridiopore. $\mathrm{Bar}=50 \mu \mathrm{m}$.

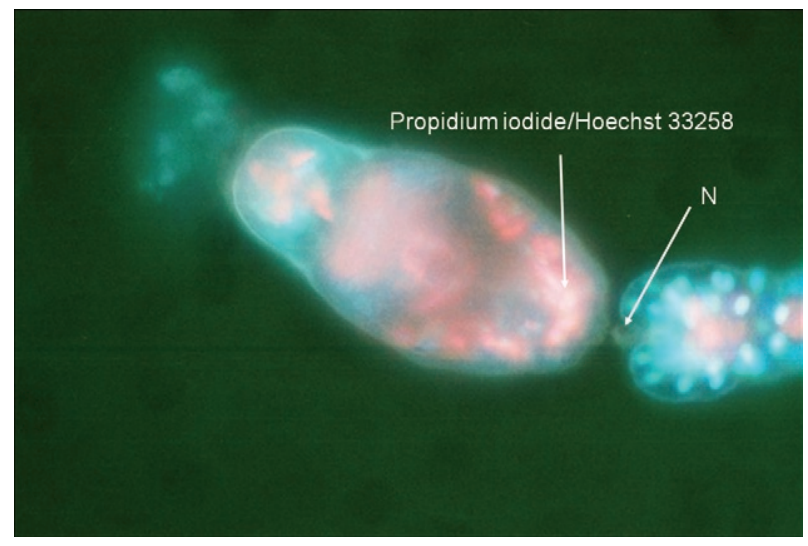

Fig. 5: living cercariae were placed in Hoechst 33258 and Propidium iodide and observed under the fluorescence microscope. The tail of the cercaria can become detached during the observation period. The concentration of the dyes near the nephridiopore and in the posterior of the parasite is shown. Bar $=50 \mu \mathrm{m}$.

parasite in a similar fashion to Lucifer Yellow (data not shown). These molecules may enter the parasite through the nephridiopore or the surface membrane. This entry of membrane impermeant molecules may have considerable implications. If skin-derived amino acids (Meyer et al. 1991) enter the parasite, they may have a nutritive function. Skin-derived growth factors have a molecular weight $(6-22 \mathrm{kDa})$ that might enable them to enter the parasite. Epidermal growth factor (Shirakata et al. 2003), keratinocyte growth factor (Escámez et al. 2004) and insulinlike growth factor-1 (Jeschke \& Herndon 2007, Todorovic et al. 2008), following entry into the parasite, may activate receptors in the parasite and affect its growth and development. Receptors for all these growth factors are 
present in the schistosome genome (www.schistoDB.net). We envision a scenario in which growth factors from the skin enter the tissues of the schistosomulum and stimulate changes in tegument metabolism and synthesis. This hypothesis can be tested by comparing the transcriptome and proteome of in vitro-generated and skin-derived schistosomula and examine the changes in signalling pathways (Dissous et al. 2007, Loverde et al. 2007, Walker \& Rollinson 2008).

\section{ACKNOWLEDGEMENTS}

John Kusel thanks the Leverhulme Trust for a travelling grant to attend the XI Symposium of Schistosomiasis honouring Dr. Piraja da Silva.

\section{REFERENCES}

Bahia D, Mortara RA, Kusel JR, Andrade LF, Ludolf F, Kuser PR, Avelar L, Trolet J, Dissous C, Pierce RJ, Oliveira G 2007. Schistosoma mansoni: expression of Fes-like tyrosine kinase SmFes in the tegument and terebratorium suggests its involvement in host penetration. Exp Parasitol 116: 225-232.

Colley DG, Wikel SK 1974. Schistosoma mansoni: simplified method for the production of schistosomules. Exp Parasitol 35: 44-51.

Dissous C, Ahier A, Khayath N 2007. Protein tyrosine kinases as new potential targets against human schistosomiasis. Bioessays 29: $1281-1288$.

Escámez MJ, García M, Larcher F, Meana A, Muñoz E, Jorcano JL, Del Río M 2004. An in vivo model of wound healing in genetically modified skin-humanized mice. $J$ Invest Dermatol 123: 1182-1191.

Grabe K, Haas W 2004. Navigation within host tissues: Schistosoma mansoni and Trichobilharzia ocellata schistosomula respond to chemical gradients. Int J Parasitol 34: 927-934.

Haas W, Diekhoff D, Koch K, Schmalfuss G, Loy C 1997. Schistosoma mansoni cercariae: stimulation of acetabular gland secretion is adapted to the chemical composition of mammalian skin. J Parasitol 83: 1079-1085.

Haas W, Grabe K, Geis C, Päch T, Stoll K, Fuchs M, Haberl B, Loy C 2002. Recognition and invasion of human skin by Schistosoma mansoni cercariae: the key-role of L-arginine. Parasitology 124: 153-167.
Howells RE, Gerken SE, Ramalho-Pinto FJ, Kawazoe U, Gazzinelli G, Pellegrino J 1975. Schistosoma mansoni: tail loss in relation to permeability changes during cercaria-schistosomulum transformation. Parasitology 71: 9-18.

Jeschke MG, Herndon DN 2007. The combination of IGF-I and KGF cDNA improves dermal and epidermal regeneration by increased VEGF expression and neovascularization. Gene Ther 14: $1235-1242$.

Loverde PT, Osman A, Hinck A 2007. Schistosoma mansoni: TGFbeta signaling pathways. Exp Parasitol 117: 304-317.

McLaren DJ, Hockley DJ 1976. Schistosoma mansoni: the occurrence of microvilli on the surface of the tegument during transformation from cercaria to schistosomulum. Parasitology 73: 169-187.

Meyer W, Poehling HM, Neurand K 1991. Intraepidermal distribution of free amino acids in porcine skin. J Dermatol Sci 2: 383-392.

Shirakata Y, Tokumaru S, Yamasaki K, Sayama K, Hashimoto K 2003. So-called biological dressing effects of cultured epidermal sheets are mediated by the production of EGF family, TGF-beta and VEGF. J Dermatol Sci 32: 209-215.

Skelly PJ, Stein LD, Shoemaker CB 1993. Expression of Schistosoma mansoni genes involved in anaerobic and oxidative glucose metabolism during the cercaria to adult transformation. Mol Biochem Parasitol 60: 93-104.

Stirewalt MA, Minnick DR, Fregeau WA 1966. Definition and collection in quantity of schistosomules of Schistosoma mansoni. Trans $R$ Soc Trop Med Hyg 60: 352-360.

Tan HH, Thornhill JA, Al-Adhami BH, Akhkha A, Kusel JR 2003. A study of the effect of surface damage on the uptake of Texas Red-BSA by schistosomula of Schistosoma mansoni. Parasitology 126: 235-240.

Todorovic V, Pesko P, Micev M, Bjelovic M, Budec M, Micic M, Brasanac D, Ilic-Stojanovic O 2008. Insulin-like growth factor-I in wound healing of rat skin. Regul Pept 150: 7-13.

Walker AJ, Rollinson D 2008. Specific tyrosine phosphorylation induced in Schistosoma mansoni miracidia by haemolymph from schistosome susceptible, but not resistant, Biomphalaria glabrata. Parasitology 135: 337-345. 